\title{
Surface charge deposition inside a capillary glass tube by an atmospheric pressure discharge in air
}

Jaroslav Jánský and Anne Bourdon

EM2C Laboratory, UPR 288 CNRS, Ecole Centrale Paris, Grande voie des vignes, 92295 Châtenay-Malabry Cedex, France

Received: date / Revised version: date

\begin{abstract}
This paper presents simulations of the dynamics of surface charging by an air plasma discharge at atmospheric pressure initiated by a needle anode inside a capillary glass tube. During the discharge propagation in the tube, the highest positive surface charge density is observed close to the point electrode. We have shown that during the discharge propagation, the positive surface charge is increasing behind the discharge front, while the electric field at the surface is decreasing. Then, we have studied the influence of the tube radius, its permittivity and the applied pulsed voltage on surface charges. We have shown that the surface charge density during the discharge propagation is inversely proportional to the tube radius and surface charge densities of $30-50 \mathrm{nC} / \mathrm{cm}^{2}$ for a tube with $R_{\text {tube }}=100 \mu \mathrm{m}$ and an applied voltage of $12 \mathrm{kV} / \mathrm{cm}$ have been obtained. We have also noted that a higher permittivity results in a higher surface charge density and a faster surface charge deposition. Then we have shown that the surface charge deposited is proportional to the applied voltage. Finally, at the end of the voltage pulse, our simulations indicate that the positive surface charge deposited during the discharge propagation in the tube decreases to very low values in few nanoseconds.
\end{abstract}

\section{Introduction}

In recent years, there has been an increasing interest for processes using atmospheric pressure electrical discharges inside random or organized two-phase media such as porous solids, monoliths or foams for various applications as flue gas treatment and chemical synthesis [1-3]. Atmospheric pressure plasmas are also routinely used to functionalize surfaces of polymers with surface roughness ranging from hundreds of nanometers to tens of micrometres [4].

In dielectric barrier discharges, surface charges deposited on dielectrics may have a significant influence on the discharge propagation as in surface dielectric barrier discharges studied for flow actuators [5-7]. In the work of 
Soloviev [6] the profile of surface charge density for a positive discharge has been calculated. A maximum value of the order of $20 \mathrm{nC} / \mathrm{cm}^{2}$ has been obtained which appeared to be in good agreement with experiments [8]. Recently, the influence of surface charges on the breakdown of surface discharges has been shown in Allegraud et al. [9].

Using dust pattern techniques, Murooka et al. [10] have shown that charge distribution on dielectric surface can be stable for several minutes at least. For a sinusoidal applied voltage, the important role of surface charges deposited during previous voltage periods on the uniformity of the discharge obtained in a plane-plane dielectric barrier discharge configuration was pointed out by Massines et al.[11,12]. In Opaits et al. [13], the influence of the constant bias voltage on the value of the deposited surface charge by successive voltage pulses of a surface dielectric barrier discharge has been shown.

In this work, we propose to study the influence of surface charges on discharges propagating in spatially confined geometries such as microcavities and pores of porous media, monoliths or foams. As a first step to a better understanding of atmospheric pressure discharge phenomena in porous materials, we propose to study an air plasma discharge at atmospheric pressure in a capillary glass tube. Recently, we have studied the influence of a radial geometrical constraint on the discharge dynamics in air at atmospheric pressure for an constant applied voltage in the range $6-9 \mathrm{kV}$ for a dielectric tube of permittivity $\varepsilon_{\mathrm{r}}$ in the range $1-10$ and tube inner radius in the range $100-600 \mu \mathrm{m}[14]$. The objective of this work is to study the discharge dynamics in conditions closer to experiments carried out in LPGP, Orsay [15] in considering an higher applied voltage in the range $12-15 \mathrm{kV}$ with a pulsed voltage. In [14], we have studied mainly the influence of the tube radius and permittivity on the discharge structure and its propagation velocity. In this work, we propose to focus on the interaction of the discharge with the surface of the capillary tube surface and the influence of the charging of the tube on the discharge propagation.

In section 2 we present the studied set-up and the discharge model. In section 3.1 we present discharge simulations and current calculations for capillary glass tube of radii $R_{\text {tube }}=100$ and $250 \mu \mathrm{m}$. In section 3.2 , the profile of surface charge density as a function of the inner tube radius, the tube permittivity and the applied voltage is discussed. Finally, in section 3.3 the time evolution of surface charge during the different phases of voltage pulse is studied.

\section{Model description}

The studied configuration is identical to that described in [14] and is only briefly summarized here. A metallic point anode on a metallic plane holder is set at $0.5 \mathrm{~cm}$ from a metallic cathode plane. The tip of the point is a semisphere with a radius of curvature of $25 \mu \mathrm{m}$. The point is immersed in a $1 \mathrm{~cm}$ long and $0.1 \mathrm{~cm}$ thick capillary glass tube. The relative permittivity of the glass tube is assumed to be $\varepsilon_{\mathrm{r}}=5$. In this work, the inner radius varies between $R_{\text {tube }}=100$ and $250 \mu \mathrm{m}$. In the experiments, the applied voltage is a single positive high voltage square 
pulse of $6-18 \mathrm{kV}$ applied at the tip. In [14], constant voltages applied at the beginning of the discharge simulation have been studied for voltages in the range $6-9 \mathrm{kV}$. In this work, we have considered higher voltages in the range $12-15 \mathrm{kV}$. To be closer to experiments, we have modelled the voltage pulse shape using a linear combination of two sigmoid functions (i.e. a smooth function with steep rise or decrease and plateau), and then the applied voltage $U_{\text {app }}$ is given by:

$$
\begin{aligned}
U_{\text {app }}(t) & =U_{\mathrm{bc}}+U_{\max }\left(\frac{1}{1+\exp \left(-\lambda\left(t-t_{\text {shift }}\right)\right)}\right. \\
& \left.+\frac{1}{1+\exp \left(\lambda\left(t-t_{\text {shift }}-t_{\text {plateau }}-t_{\text {rise }}\right)\right)}\right),
\end{aligned}
$$

where $U_{\mathrm{bc}}=100 \mathrm{~V}$ is a small constant applied voltage $U_{\max }$ is the maximum of the sigmoidal function (e.g. $11.9 \mathrm{kV}$ for a $12 \mathrm{kV}$ applied voltage), $t_{\text {shift }}=2 \mathrm{~ns}, \lambda=8 / t_{\text {rise }}$ where $t_{\text {rise }}=2 \mathrm{~ns}$ is the rise and decrease time of the voltage and $t_{\text {plateau }}=5 \mathrm{~ns}$ is the duration of the voltage plateau.

As in [14], a classical fluid model based on the drift-diffusion equations for electrons and ions coupled with Poisson's equation is used to simulate the discharge propagation. To take into account the different dielectric permittivities in air and in the dielectric tube, we use Poisson's equation with variable coefficients :

$$
\begin{gathered}
\frac{\partial n_{\mathrm{e}}}{\partial t}-\boldsymbol{\nabla} \cdot\left(n_{\mathrm{e}} \mu_{\mathrm{e}} \boldsymbol{E}\right)-\boldsymbol{\nabla} \cdot\left(D_{\mathrm{e}} \boldsymbol{\nabla} n_{\mathrm{e}}\right)=S_{\mathrm{ph}}+S_{\mathrm{e}}^{+}-S_{\mathrm{e}}^{-} \\
\frac{\partial n_{\mathrm{p}}}{\partial t}+\boldsymbol{\nabla} \cdot\left(n_{\mathrm{p}} \mu_{\mathrm{p}} \boldsymbol{E}\right)=S_{\mathrm{ph}}+S_{\mathrm{p}}^{+}-S_{\mathrm{p}}^{-} \\
\frac{\partial n_{\mathrm{n}}}{\partial t}-\nabla \cdot\left(n_{\mathrm{n}} \mu_{\mathrm{n}} \boldsymbol{E}\right)=S_{\mathrm{n}}^{+}-S_{\mathrm{n}}^{-} \\
\nabla \cdot(\varepsilon \nabla V)=-q_{\mathrm{e}}\left(n_{\mathrm{p}}-n_{\mathrm{n}}-n_{\mathrm{e}}\right)-\sigma \delta_{\mathrm{s}}
\end{gathered}
$$

where subscripts "e", "p" and "n" refer to electrons, positive and negative ions, respectively, $n_{i}$ is the number den- sity of species $i, V$ is the potential, $\boldsymbol{E}=-\boldsymbol{\nabla} V$ is the electric field, $\mu_{i}$ is the absolute value of the mobility of species $i$ and $D_{\mathrm{e}}$ is the diffusion coefficient of electrons. On timescales of interest for studies presented in this paper, diffusion of ions is neglected. The $S^{+}$and $S^{-}$terms stand for the rates of production and loss of charged particles. The $S_{\mathrm{ph}}$ term is the rate of electron-ion pair production due to photoionization in a gas volume. Transport parameters and reaction rates are taken from [16]. For the photoionization source term, the three-group $\mathrm{SP}_{3}$ model is used [17] with boundary conditions given in [18]. In Eq. (5), $q_{\mathrm{e}}$ is the absolute value of electron charge, $\varepsilon=\varepsilon_{\mathrm{r}} \varepsilon_{0}$ is the permittivity with $\varepsilon_{0}$ the vacuum permittivity and $\varepsilon_{\mathrm{r}}$ the relative permittivity $\left(\varepsilon_{\mathrm{r}}=1\right.$ in the air discharge in the tube and in air around the tube and $\varepsilon_{\mathrm{r}}=5$ inside the dielectric tube). $\sigma \delta_{\mathrm{s}}$ represents the contribution of the charges deposited by the discharge on the dielectric surface. These charges are obtained by time integrating charged particle fluxes to the surface. In this work, boundary conditions are the same as in [14]. At the dielectric surface, we have considered secondary emission of electrons by ion bombardment (with a secondary emission coefficient of 0.1 ). The use of this high value is a way to take into account roughly other secondary emission processes as photoemission, secondary emission of electrons by impact of metastable molecules and field emission.

In this paper, axisymmetric discharges are studied and thus cylindrical coordinates $(x, r)$ are used with the $x$-axis as axis of the discharge. The tip of the anode point is located at $x=0.5 \mathrm{~cm}$ and the grounded cathode is located 
at $x=0$. We have used a $1 \times 2.5 \mathrm{~cm}^{2}$ computational domain. The grid is Cartesian, with a mesh size of $0.5 \mu \mathrm{m}$ at the electrode tip. This small mesh is needed to ensure the numerical stability during the voltage decrease. In the axial direction, the mesh size is expanded towards the cathode (for $x \leq 0.48 \mathrm{~cm}$ ) following a geometric progression until it reaches $5 \mu \mathrm{m}$ and then is kept constant. Towards the plane holder (for $x \geq 0.51 \mathrm{~cm}$ ) the mesh size is expanded following a geometric progression. In the radial direction, the mesh size is $0.5 \mu \mathrm{m}$ from the symmetry axis up to $r=110 \mu \mathrm{m}$ and then expanded following a geometric expansion. As an example, the final rectilinear grid for the whole computational domain for the condition of Fig. 1 with a capillary tube of $R_{\text {tube }}=100 \mu \mathrm{m}$ is a grid with $n_{x} \times n_{r}=1626 \times 380$ cells.

The charged species transport equations are solved using the improved Scharfetter-Gummel algorithm [19] with linear field approximation (ISG-1). A detailed discussion on the influence of the value of $\epsilon_{\mathrm{ISG}}$, the parameter of this numerical scheme, is given in [14]. In this work, we have used the same value of $\epsilon_{\mathrm{ISG}}=0.1$ as in [14] for tubes with $\varepsilon_{\mathrm{r}}=5$. Poisson's equation is discretized using a finite volume approach over the whole computational domain. At the metallic electrode-air interfaces, the ghostfluid method is used [20]. One-sided 2nd order derivatives are used for discretizing the electric field on the tube interface. In this case, it is important to note that the resulting matrix is no more symmetric positive definite. The resulting linear system is solved using the direct solver MUMPS [21].
In this paper, we have calculated the discharge current flowing in the external circuit. Two methods have been used. First, the current can be derived from the integration of the total current flux over a surface parallel to the plane cathode:

$$
I=\int_{S} \boldsymbol{J} \cdot \mathbf{d} \boldsymbol{S}
$$

where $\mathrm{S}$ is a surface described by equation $\{x=$ Const $\}$ and the total current flux $\boldsymbol{J}$ is given by:

$$
\boldsymbol{J}=\boldsymbol{j}+\varepsilon \frac{\partial \boldsymbol{E}}{\partial t}
$$

where $\boldsymbol{j}$ is the conduction current. As the total current is conserved, if we consider the current calculated using Eq. (6) through two different surfaces located at $x_{1}$ and $x_{2}$, the difference between the two currents is equal to the current flux at the radial boundary $r=R_{\max }$ of the computational domain. In this work, we have used a Neumann boundary condition for the electric field at the radial boundary $r=R_{\max }$ which ensures that the capacitive current at the boundary is equal to zero. We have also checked that the currents calculated with Eq. (6) for two surfaces $\{x=0.2 \mathrm{~cm}\}$ and $\{x=0.4 \mathrm{~cm}\}$ are equal with a relative error of the order of $10^{-6}$.

Second, the current can be derived from the energy balance equation (equation 14 in [22]):

$$
I=\frac{1}{U_{\text {app }}} \int_{V} \boldsymbol{J} \cdot \boldsymbol{E} \mathrm{d} V,
$$

where $V$ is the volume of the computational domain. For a discharge between metallic electrodes without dielectric surfaces, the general expression (Eq. 8) can be simplified to obtain the well known Sato's equation for time dependent applied voltages (equation 24 in [22]). In Sato's equa- 
tion, the Laplacian electric field and its time derivative are used. For a discharge between metallic electrodes, the Laplacian field is proportional to applied voltage and then it can be calculated only once at the beginning of the simulation and used to easily calculate the current at any time.

For dielectric barrier discharges, it is possible to simplify Eq. (8) for the studied geometry as was done in [23] for a plane-plane dielectric barrier discharge and to express the current as a function of the Laplacian electric field (taking into account surface charges) and its time derivative. However, it is important to note that even for this simple geometry, the obtained expression is much more complex than the Sato's equation. Furthermore, with surface charge deposition during the discharge propagation, the spatial distribution of the Laplacian field varies in time and has to be recalculated at each time step.

As in discharge simulations we compute directly the total electric field at each time step, for discharges with presence of dielectrics as the one studied in this work, it is then more convenient to use directly Eqs. (6) and (8) to compute the discharge current. For both equations, the time derivative of the total electric field has to be calculated accurately to determine the total current flux in Eq. (7). In this work, we have noted that the use of an iterative solver (as the NAG routine used in [20]) for Poisson's equation does not allow to have an accurate calculation of the capacitive current unless a very high accuracy is demanded (the error on the electric field calculation has to be much less than the change of electric field during one time step). Conversely, for a direct solver as the MUMPS [21] solver used in this work, the accuracy of the solution is determined from the roundoff error of computer and from the type of variables. The use of double precision gives a relative error on the electric field calculation of the order of $10^{-14}$, which allows an accurate calculation of the current.

\section{Results}

\subsection{Discharge propagation in glass tubes with}

$R_{\text {tube }}=100$ and $250 \mu \mathrm{m}$

In this section we present simulation results of the discharge propagation in capillary glass tubes in air at atmospheric pressure for two values of the tube inner radius $R_{\text {tube }}=100$ and $250 \mu \mathrm{m}$ for an applied voltage $U_{\text {app }}=$ $12 \mathrm{kV}$. Fig. 1 shows the distributions of electron density, electric field during the discharge propagation at $t=4.0$ and $5.5 \mathrm{~ns}$ for a tube with an inner radius of $R_{\text {tube }}=$ $100 \mu \mathrm{m}$. Axial profiles on the tube surface of the surface charge density, and the total electric field at the tube interface are also shown. We have checked that the propagation of the discharge in the tube is stable and then as shown on Fig. 1, the discharge front structure is the same for both time moments. These results are consistent with the results obtained for lower applied voltages in [14]. In the vicinity of the discharge front, a high electric field and a low electron density are obtained in the sheath layer close to the tube. This sheath region disappears about $0.1 \mathrm{~cm}$ behind the discharge front when positive ions have suffi- 


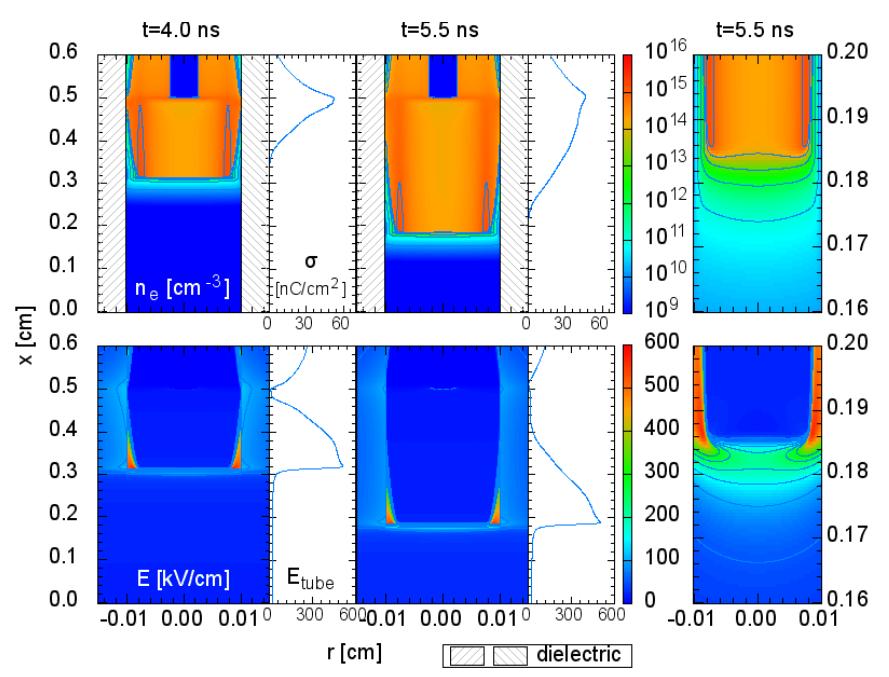

Fig. 1. Cross-sectional views of the electron density and the magnitude of the electric field during discharge propagation in a glass tube with $R_{\text {tube }}=100 \mu \mathrm{m}$ for an applied voltage $U_{\text {app }}=$ $12 \mathrm{kV}$ at $t=4$ and $5.5 \mathrm{~ns}$. For both times, the axial profiles on the tube surface of $\sigma$, the surface charge density, and $E_{\text {tube }}$ the total electric field at the tube interface are shown. Last figure of each line shows the discharge front in proportional scale at $t=5.5 \mathrm{~ns}$.

ciently charged the surface to shield the electric field. This is clearly shown on the axial profiles of the surface charge and the total electric field at the tube interface in Fig. 1. Indeed, behind the discharge front during its propagation, the surface charge is increasing while the electric field at the surface is decreasing.

To study more in detail the interaction of the discharge with the capillary tube, Fig. 2 shows for the conditions of Fig. 1, the radial profiles of electron and positive ion densities and of the electric field at $x=0.4 \mathrm{~cm}$ and $t=4$ and 5.5 ns. As shown on Fig. 1, at $t=4$ ns, the discharge front has already passed the considered section $x=0.4 \mathrm{~cm}$ and the sheath is formed. Fig. 2 shows that the electron and

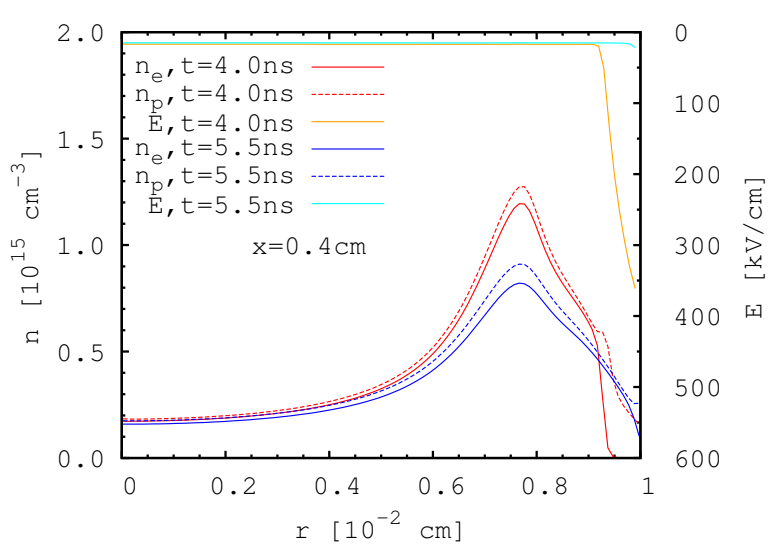

Fig. 2. Radial profiles of electron and positive ion densities and of the total electric field at $x=0.4 \mathrm{~cm}$ for the conditions of Fig. 1.

positive ion densities are very close for $r \leq 90 \mu \mathrm{m}$, which corresponds to a low electric field. The electron density decreases steeply at $r=90 \mu \mathrm{m}$ and is very low close to the tube surface. Conversely, the density of positive ions is much higher than the electron density close to the surface for $r \geq 90 \mu \mathrm{m}$ and then the electric field increases rapidly to reach a high value of $400 \mathrm{kV} / \mathrm{cm}$ at the tube surface. The electric field is directed towards the dielectric surface and then electrons are emitted from the tube surface by secondary emission due to positive ions. Furthermore positive ions charge the tube surface to shield the electric field. Then, only $1.5 \mathrm{~ns}$ later, at $t=5.5 \mathrm{~ns}$, Fig. 2 shows that the electron density close to the tube surface is much higher than the one observed at $t=4.0 \mathrm{~ns}$ and is close to the positive ion density for $r \leq R_{\text {tube }}$. Then, the electric field is shielded and is almost zero for $r \leq R_{\text {tube }}$.

Figure 3 shows the discharge current flowing in the external circuit as a function of time for the discharge conditions of Fig. 1. First, we have checked that the two meth- 


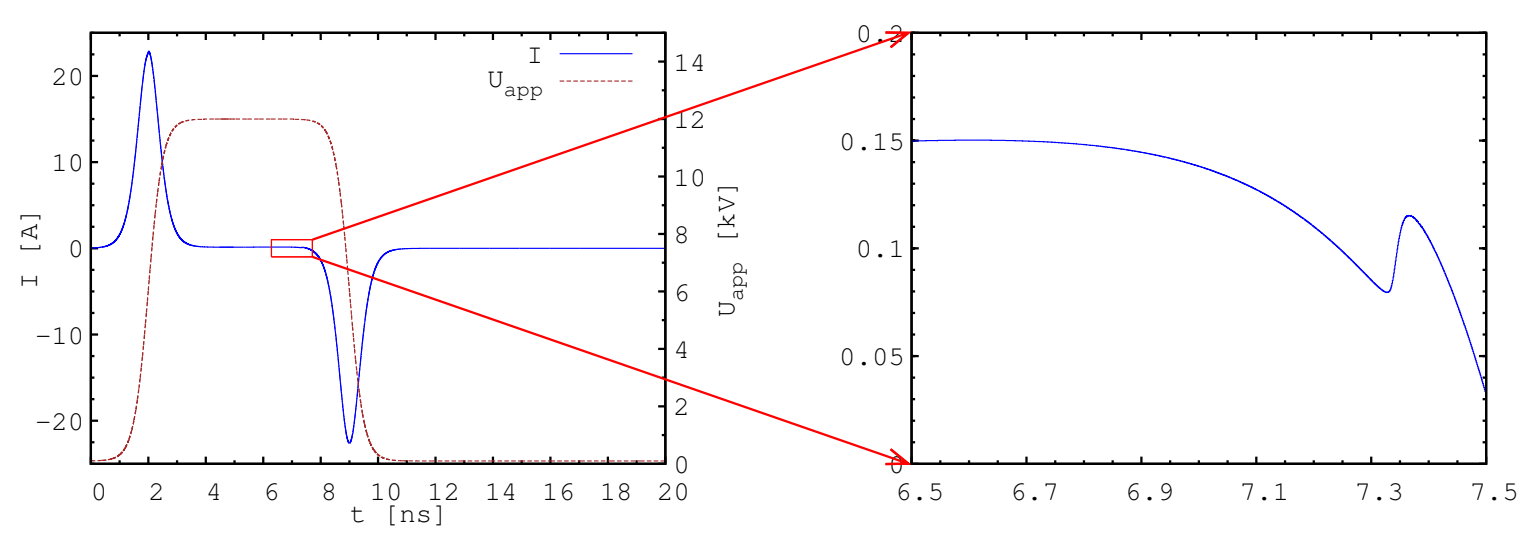

Fig. 3. Time evolution of the applied voltage and total current for the conditions of Fig. 1.

ods to calculate the current (Eqs. (6) and (8)) give the same result with a relative error of order $10^{-2}$. From a computational point of view, the calculation of the current using Eq. (6) on a given surface is faster than the calculation of the volume integral of Eq. (8). Then, in the following we have used Eq. (6) to calculate the discharge current. On Fig. 3, the first current peak at $t=2 \mathrm{~ns}$ corresponds to the capacitive current due to the voltage rise. The current resulting from the expansion phase of the discharge is much smaller in amplitude and is hidden in this first peak of capacitive current. The right part of the figure shows a zoom of the time interval 6.5 to $7.5 \mathrm{~ns}$. The current peak observed at $t=7.3 \mathrm{~ns}$ corresponds to the impact of the discharge on the grounded plane electrode. In the following sections, we have used this current peak to determine the time of impact of the discharge on the grounded plane electrode for all the simulated discharge conditions. On Fig. 3, it is important to note that due to the decrease of the applied voltage, the total current is already decreasing at $t=7.3 \mathrm{~ns}$, when the discharge impacts the grounded electrode. At $t=9 \mathrm{~ns}$, a second negative ca-

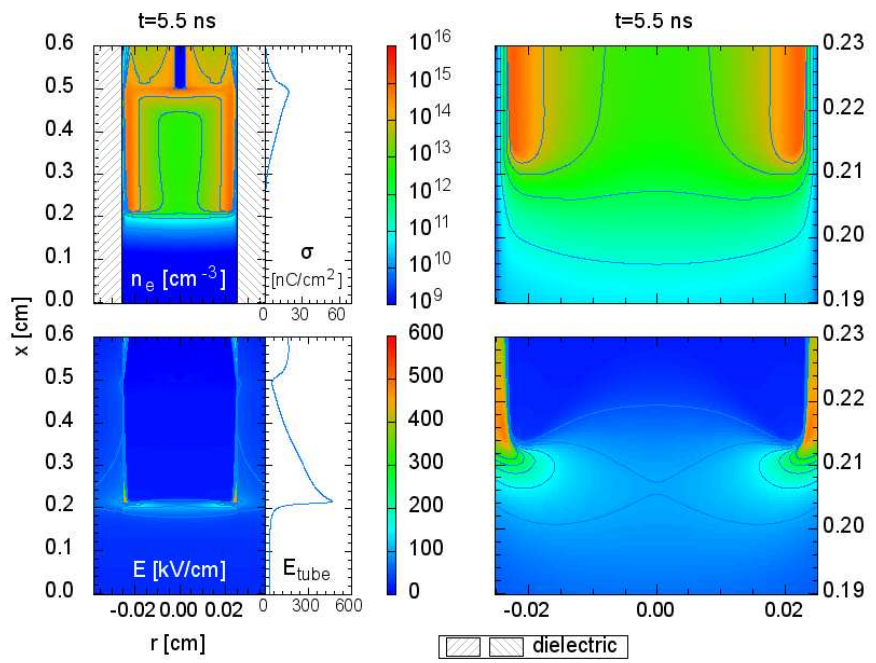

Fig. 4. Cross-sectional views of the electron density and the magnitude of the electric field during discharge propagation in a glass tube with $R_{\text {tube }}=250 \mu \mathrm{m}$ for an applied voltage $U_{\text {app }}=12 \mathrm{kV}$ at $t=5.5 \mathrm{~ns}$. Last figure of each line shows the discharge front in proportional scale. The axial profiles on the tube surface of $\sigma$, the surface charge, and $E_{\text {tube }}$ the total electric field at the tube interface are also shown.

pacitive peak is observed due to the voltage decrease. After the voltage decrease, a small constant voltage of $100 \mathrm{~V}$ is applied (Eq. (1)) and the discharge current is equal to $3 \mathrm{~mA}$.

Figure 4 shows the distributions of electron density, elec- 
tric field during the discharge propagation at time $t=$ $5.5 \mathrm{~ns}$ for a tube with an inner radius of $R_{\text {tube }}=250 \mu \mathrm{m}$ for the same applied voltage as in Fig. 1. Axial profiles on the tube surface of the surface charge density, and the total electric field at the tube interface are also shown. For an applied voltage of $12 \mathrm{kV}$, we note that the discharge is more tubular for $R_{\text {tube }}=250 \mu \mathrm{m}$ than for $R_{\text {tube }}=$ $100 \mu \mathrm{m}$, in agreement with results obtained at lower voltages [14]. It is also interesting to point out that the peak value of the electric field in the discharge front and in the sheath region are smaller for $R_{\text {tube }}=250 \mu \mathrm{m}$ than for $R_{\text {tube }}=100 \mu \mathrm{m}$. Conversely, the sheath thickness at the vicinity of the discharge front is almost the same. As a consequence, the shielding process behind the discharge front is slower for the case of $R_{\text {tube }}=250 \mu \mathrm{m}$ and then the decrease of the electric field at the tube interface for $R_{\text {tube }}=250 \mu \mathrm{m}$ is smaller than the one observed on Fig. 1 for $R_{\text {tube }}=100 \mu \mathrm{m}$.

\subsection{Parametric study of the deposited surface charge} on the dielectric surface during the discharge propagation

In this section, we study the influence of the radius of the tube, its relative permittivity and of the applied voltage, on the deposited surface charges on the dielectric surface. First, the axial profiles of surface charges on Figs. 1 and 4 show that the surface charge density is higher for smaller radius. To compare more in detail the results for both radii, we have calculated the linear charge density $\tau$ which

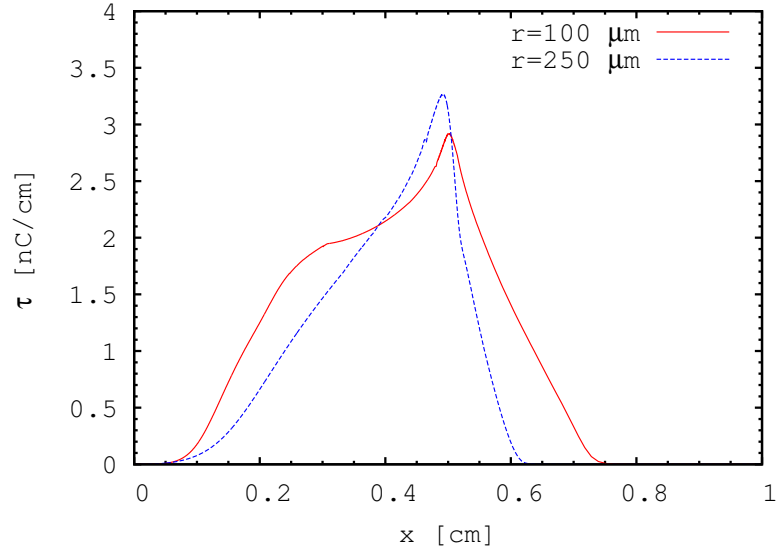

Fig. 5. Axial profile of the linear charge density on the surface of the dielectric glass tube of radius $R_{\text {tube }}=100$ and $250 \mu \mathrm{m}$ at $t=7.2$ ns (i.e. just before the discharge impact on the cathode plane for both radii). The applied voltage is $U_{\text {app }}=12 \mathrm{kV}$.

is the total charge per unit length in the axial direction:

$$
\tau=\sigma 2 \pi r
$$

Figure 5 shows the axial profile of the linear charge density for $R_{\text {tube }}=100$ and $250 \mu \mathrm{m}$ at $t=7.2$ ns (i.e. just before the discharge impact on the cathode plane for both radii). In both cases, the maximum of the surface charge is located close to the point electrode at $x=0.5 \mathrm{~cm}$ and the surface charge decreases for $x<0.5 \mathrm{~cm}$ and $x>0.5 \mathrm{~cm}$. On Fig. 5, we note that the radius of the tube has a weak influence of the linear charge density deposited on the tube during the discharge propagation. We note also that the slope of the linear charge density on the grounded electrode side (e.g. at $x=0.2 \mathrm{~cm}$ ) is steeper for a smaller radius, corresponding to a faster shielding process as was discussed in the previous section. Figure 6 shows the influence of the permittivity of the tube on the axial profile of surface charge density just before the discharge impact on the cathode plane for a capillary tube with 


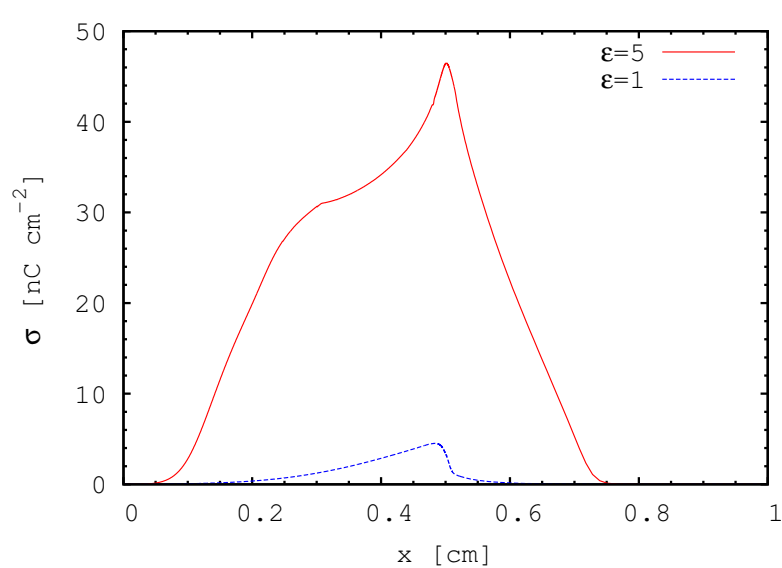

Fig. 6. Axial profile of the surface charge on a capillary tube with $\varepsilon_{\mathrm{r}}=1$ and 5 and $R_{\text {tube }}=100 \mu \mathrm{m}$ for an applied voltage $U_{\text {app }}=12 \mathrm{kV}$ just before the discharge impact on the grounded plane (i.e. at $t=7.2 \mathrm{~ns}$ for $\varepsilon_{\mathrm{r}}=5$ and $t=5.8 \mathrm{~ns}$ for $\varepsilon_{\mathrm{r}}=1$ ).

$R_{\text {tube }}=100 \mu \mathrm{m}$. We compare results obtained for $\varepsilon_{\mathrm{r}}=1$ and 5. As already observed at lower voltages in [14], the discharge is faster for smaller values of the tube permittivity. Then on Fig. 6, we compare the surface charge density at $t=5.8 \mathrm{~ns}$ for $\varepsilon_{\mathrm{r}}=1$ and $t=7.2 \mathrm{~ns}$ for $\varepsilon_{\mathrm{r}}=5$. For permittivity $\varepsilon_{\mathrm{r}}=1$, the surface charge density is significantly smaller than for $\varepsilon_{\mathrm{r}}=5$. A higher permittivity corresponds to an enhanced radial electric field at the tube interface and then in the sheath. Consequently, the charge deposited at the tube surface has to be higher to shield it. It is interesting to note that the shielding time for $\varepsilon_{\mathrm{r}}=1$ is longer than for $\varepsilon_{\mathrm{r}}=5$. For our simulations at $\varepsilon_{\mathrm{r}}=1$, we have observed that for all points of the tube surface, the electric field at the tube surface is not shielded during the discharge propagation and even at the arrival of the discharge at the grounded electrode.

Then we have studied the influence of the applied voltage on the axial profile of the surface charge density just before

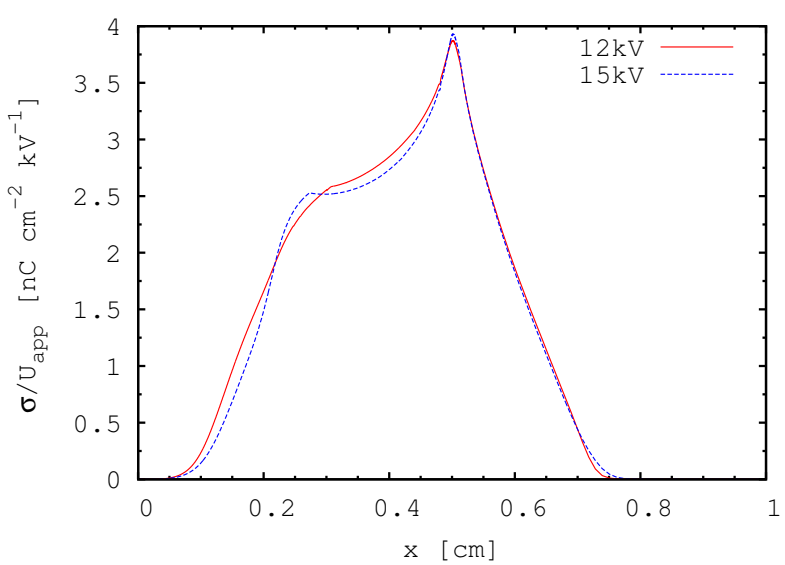

Fig. 7. Axial profile of $\sigma / U_{\text {app }}$ (the surface charge density divided by the applied voltage) on a capillary glass tube for a tube radius of $R_{\text {tube }}=100 \mu \mathrm{m}$ and two applied voltages $U_{\text {app }}=12$ and $15 \mathrm{kV}$ just before the discharge impact on the grounded plane (i.e. $t=7.2 \mathrm{~ns}$ for $12 \mathrm{kV}$ and $t=5.6 \mathrm{~ns}$ for $15 \mathrm{kV})$.

the discharge impact on the cathode plane for a capillary glass tube with $R_{\text {tube }}=100 \mu \mathrm{m}$. Figure 7 shows the axial profile of $\sigma / U_{\text {app }}$ (the surface charge density divided by the applied voltage) for two values of applied voltages 12 and $15 \mathrm{kV}$. As already observed at lower voltages in [14], the propagation velocity of the discharge increases as the voltage increases. Then on Fig. 7, we compare the values of $\sigma / U_{\text {app }}$ at $t=5.6 \mathrm{~ns}$ for $U_{\text {app }}=15 \mathrm{kV}$ and at $t=7.2 \mathrm{~ns}$ for $12 \mathrm{kV}$. We note that both curves are almost identical and then the surface charge density deposited during the discharge propagation increases as the applied voltage increases. In fact, the voltage and surface charge density needed to shield the electric field at the tube interface are linearly dependent on the electric field. Then if the applied voltage is increased by a given factor then the surface charge density is multiplied by the same factor. 


\subsection{Study of the time evolution of the deposited} surface charge on the dielectric surface

In this section, we study the time evolution of the deposited surface charge as a function of time during the whole duration of the voltage pulse at two different positions on the dielectric tube surface: $x=0.5 \mathrm{~cm}$ (located at the point electrode) and $x=0.4 \mathrm{~cm}$. We consider the same conditions as Fig. 1, with a glass tube of radius $R_{\text {tube }}=100 \mu \mathrm{m}$ and an applied voltage $U_{\text {app }}=12 \mathrm{kV}$. For the two studied positions, Fig. 8 shows the time evolutions of the surface charge density, the applied voltage and the radial electric field at the tube interface. When the discharge passes the position $x=0.4 \mathrm{~cm}$ at $t=3 \mathrm{~ns}$, an increase of the radial electric field is observed and the charging of the tube starts. The surface charge increases up to $35 \mathrm{nC} . \mathrm{cm}^{-2}$ at $t=6 \mathrm{~ns}$ which corresponds to the time when the electric field is shielded at the tube surface. Then the electric field becomes slightly negative which allows the electrons to impact the tube and then the surface charge slowly decreases. The mobility of electrons for low electric field (few $\mathrm{kV} / \mathrm{cm}$ ) is three orders of magnitude higher than the mobility of positive ions, then the electrons can efficiently charge the dielectric surface. At $t=7.3 \mathrm{~ns}$ (Fig. 3), the discharge impacts the grounded electrode, and we note that this has a negligible influence on the radial electric field at the tube surface at $x=0.4 \mathrm{~cm}$. As the applied voltage decreases, an increase of the negative radial electric field is observed and consequently the surface charge density decreases rapidly in a few nanoseconds. Finally, we note that surface charge density and electric field decrease to zero at the end of the voltage pulse. At the position $x=0.5 \mathrm{~cm}$ (at the point electrode), Fig. 8 shows that the time evolutions of surface charge density and radial electric field are more complex. After the initial radial expansion of the discharge around the point, the discharge interacts with the tube at $x=0.5 \mathrm{~cm}$ and start to charge it with positive charges at $t=2.5 \mathrm{~ns}$. A peak value of the surface charge of about $50 \mathrm{nC} . \mathrm{cm}^{-2}$ is observed at $t=4 \mathrm{~ns}$. Then a rapid shielding occurs with even a small negative electric field which results in a small decrease of the surface charge. After the impact of the discharge on the cathode $(t=7.3 \mathrm{~ns})$, the redistribution of the electric field close to the point electrode results in a positive radial electric field with an increase of the surface charge density. When the applied voltage starts to decrease, the electric field changes of polarity to negative values at $t=8.5 \mathrm{~ns}$ and consequently the surface charge density decreases. The dynamics of this process is very fast and we note that even the surface charge density turns to negative values at $t=10 \mathrm{~ns}$. After the voltage pulse, a small constant voltage of $100 \mathrm{~V}$ is applied (Eq. (1)), and we note that the radial electric field becomes positive due to the negatively charged surface. This field attracts positive ions which start to neutralize the surface charge but due to the low mobility of ions this process takes a longer time than the simulation time considered in this work (20ns). On Fig. 8, it is interesting to note that the surface charge density after the voltage pulse is very small. However, it is important to note that a significant amount of volume charges are in the air in the tube 
(about $10^{14} \mathrm{~cm}^{-3}$ electrons and positive ions). Then on much longer timescales than those simulated in this work, these volume charges will recombine and diffuse towards the electrodes and the surface of the dielectric tube. The simulation of the post-discharge on long timescales will be presented in a dedicated paper. Finally, it is interesting to note that the values of positive surface charges in the range $30-50$ nC.cm ${ }^{-2}$ obtained during the propagation of the discharge in the tube are of the same order as surface charges calculated in positive planar surface discharges in air at atmospheric pressure [6].

\section{Conclusion}

In this work, we present simulations of the surface charge deposition inside a capillary glass tube during an air discharge propagation at atmospheric pressure. The discharge is initiated by a needle set in the capillary tube. A voltage pulse with $2 \mathrm{~ns}$ rise time and $5 \mathrm{~ns}$ plateau with an applied voltage in the range $12-15 \mathrm{kV}$ has been considered. First, the dynamics of the discharge propagation and the surface charge deposition for a glass tube of inner radius $R_{\text {tube }}=100 \mu \mathrm{m}$ is studied. In the vicinity of the discharge front, a high electric field and a low electron density are observed in the sheath layer close to the tube surface. Behind the discharge front, the surface charge is increasing while the electric field at the surface is decreasing. The study of the time evolutions of radial profiles of electron and positive ion densities and electric field at a given point of the tube surface, shows the dynamics of the interaction of the discharge with the glass tube. Just behind the discharge front, the density of positive ions near the tube surface is much higher than the electron density and then a high electric field is obtained close to the surface. Then as this electric field is directed towards the dielectric surface, electrons are emitted from the tube surface by secondary emission due to positive ions. Furthermore, positive ions charge the tube surface to shield the electric field. Then, rapidly (in about $1.5 \mathrm{~ns}$ ), the electric field becomes very low at the considered position and the electron density near the tube interface becomes close to the positive ion density.

For this configuration, the discharge current flowing in the external circuit has been calculated. Two significant capacitive current peaks corresponding to the increase and decrease of the applied voltage have been obtained and a small current peak corresponding to the discharge impact on the grounded electrode has been observed. In this work, we have used this current peak to determine the time of impact of the discharge on the grounded plane electrode for all the simulated discharge conditions. We have tested two methods for the current calculation and we have checked that both methods give the same results. To calculate the discharge current in a discharge with presence of dielectric material as the one studied in this work, we have checked that more accurate results are obtained using direct solver instead of iterative solver for Poisson's equation.

Then we have studied the discharge propagation in a glass tube with inner radius $R_{\text {tube }}=250 \mu \mathrm{m}$. In this case, the discharge is more tubular than for $R_{\text {tube }}=100 \mu \mathrm{m}$, in 

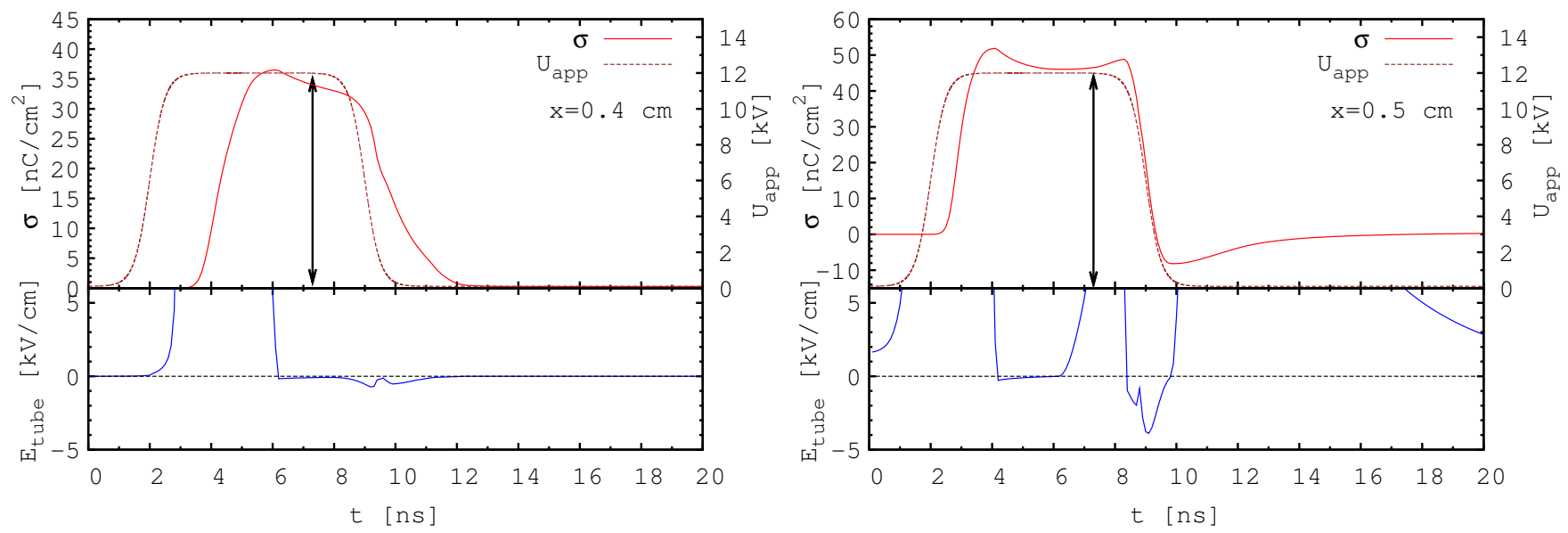

Fig. 8. Time evolutions of surface charge density, applied voltage and radial electric field at the tube surface at $x=0.4 \mathrm{~cm}$ and $x=0.5 \mathrm{~cm}$ (point electrode) for the conditions of Fig. 1. The electric field range is chosen to show mainly the changes of polarity. The vertical arrow corresponds to the impact of the discharge on the grounded electrode at $t=7.3 \mathrm{~ns}$ (Fig. 3).

agreement with results obtained at lower voltages [14]. It is also interesting to point out that the peak value of the electric field in the discharge front and in the sheath region are smaller for $R_{\text {tube }}=250 \mu \mathrm{m}$ than for $R_{\text {tube }}=100 \mu \mathrm{m}$. Conversely, the sheath thickness at the vicinity of the discharge front is almost the same. As a consequence, the shielding process behind the discharge front is slower for the case of $R_{\text {tube }}=250 \mu \mathrm{m}$ and then the decrease of the electric field at the tube interface for $R_{\text {tube }}=250 \mu \mathrm{m}$ is smaller than the one observed for $R_{\text {tube }}=100 \mu \mathrm{m}$. To compare the surface charges for both radii, we have calculated the axial profile of the linear charge density just before the discharge impact on the cathode plane. For both radii, the maximum of the surface charge is located close to the point electrode at $x=0.5 \mathrm{~cm}$ and the surface charge decreases for $x<0.5 \mathrm{~cm}$ and $x>0.5 \mathrm{~cm}$. Furthermore, we have shown that the radius of the tube has a weak influence of the linear charge density deposited on the tube during the discharge propagation. Then we have studied the influence of the permittivity of the tube on the surface charge density deposited during the discharge propagation. We have shown that a higher permittivity results in a higher surface charge density and a faster surface charge deposition. Then we have studied the influence of the applied voltage on the surface charge density deposited during the discharge propagation. We have shown that the surface charge deposited is proportional to the applied voltage. Finally, we have studied the time evolution of the surface charge during the whole voltage pulse at two different locations of the tube surface. We have shown that the discharge is very efficient to charge the tube surface during its propagation (surface charges densities up to values of $30-50 \mathrm{nC} / \mathrm{cm}^{2}$ for a tube with $R_{\text {tube }}=100 \mu \mathrm{m}$ and an applied voltage of $12 \mathrm{kV} / \mathrm{cm}$ have been obtained). It is interesting to note that the values of positive surface charges obtained during the propagation of the discharge in a capillary glass tube are of the same order as surface charges obtained in positive planar surface discharges in 
air at atmospheric pressure. Our results show that the impact of the discharge on the grounded electrode has a small influence of the surface charge. Conversely, due to the voltage decrease at the end of the voltage pulse, the positive surface charge deposited during the discharge propagation in the tube decreases rapidly (in a few nanoseconds) to very low values. Indeed during the voltage decrease, we have shown that the electric field changes of polarity to negative values and consequently electrons may impact on the surface and efficiently neutralize the positive surface charge. Then after the end of the voltage pulse, we have shown that the surface charge on the surface of the tube is very small. However, it is important to note that a significant amount of volume charges are in air inside the tube. Then on much longer timescales than those simulated in this work (20 ns) these volume charges will recombine and diffuse towards the electrodes and the surface of the dielectric tube. The resulting surface and volume charges could have an impact on the next discharge if a repetitively pulsed discharge is used. The simulation of the postdischarge on much longer timescales (up to a few seconds) will be presented in a dedicated paper.

\section{Acknowledgement}

The authors thank the Agence Nationale de la Recherche for its support of the ALVEOPLAS project (Grant No. ANR-08-BLAN-0159-01).

\section{References}

1. Hammer T, Plasma Sources Sci. Technol. 11, (2002) A196.

2. Pasquiers S, Eur. Phys. J. Appl. Phys. 28, (2004) 319-324.

3. Kim H H, Plasma Process. Polym. 1, (2004) 91-110.

4. Bhoj A N, Kushner M J, Plasma Sources Sci. Technol. 17, (2008) 035024.

5. Unfer T, Boeuf J P, J.Phys.D: Appl.Phys. 42, (2009) 194017.

6. Soloviev V R, Krivtsov V M, J.Phys.D: Appl.Phys. 42, (2009) 125208.

7. Likhanskii A V, Semak V V, Schneider M N, Opaits D F, Miles R B, Macheret S O, 47th AIAA Aerospace Sciences Meeting Including The New Horizons Forum and Aerospace Exposition, AIAA 2009-841.

8. Gibalov V I, Pietsch G J, J. Phys. D: Appl. Phys. 33, (2000) 2618-2636.

9. Allegraud K, Guaitella O, Rousseau A, J. Phys. D: Appl. Phys. 40, (2007) 7698-7706.

10. Murooka Y, Koyama S, J. Appl. Phys. 50, (1979) 62006206.

11. Massines F, Rabehi A, Decomps Ph, Gadri R B, Ségur P, Mayoux Ch, J. Appl .Phys. 83, (1998) 2950.

12. Massines F, Ségur P, Gherardi N, Khamphan C, Ricard A, Surf. and Coat. Technol. 174-175, (2003) 8-14.

13. Opaits D F, Schneider M N, Miles R B, Likhanskii A V, Macheret S O, Physics of Plasmas 15, (2008) 073505.

14. Jánský J, Tholin F, Bonaventura Z, Bourdon A, J. Phys. D: Appl. Phys. 43, (2010) 395201.

15. Le Delliou L, Tardiveau P, Jeanney P, Bauville G, Jorand F, Pasquiers S, proceedings of XVIII International Conference on Gas Discharges and Their Applications (GD 2010) 
16. Morrow R, Lowke J J, J. Phys. D: Appl. Phys. 30, (1997)

614-627.

17. Bourdon A, Pasko V, Liu N, Célestin S, Ségur P, Marode

E, Plasma Sources Sci. Technol. 16, (2007) 656-678.

18. Liu N Y, Célestin S, Bourdon A, Pasko V P, Ségur P, Marode E, Appl. Phys. Lett. 91, (2007) 211,501.

19. Kulikovsky A A, J. Comput. Phys. 119, (1995) 149-155.

20. Célestin S, Bonaventura Z, Zeghondy B, Bourdon A, Ségur P, J. Phys. D: Appl. Phys. 42, (2009) 065203.

21. Amestoy P, Duff I, L'Excellent J, Koster J, SIAM Journal of Matrix Analysis and Applications 23, (2001) 15-41.

22. Morrow R and Sato N, J. Phys. D: Appl. Phys. 32, (1999) L20-L22.

23. Potin J, Phd thesis, University Paul Sabatier, Toulouse, France, 2001. 